\title{
Rapid evolution of the functionally conserved gap gene giant in Drosophila
}

\author{
Wenhan Chang ${ }^{1}$, Daniel R. Matute ${ }^{2}$, and Martin Kreitman ${ }^{1, \mathbb{}}$ \\ ${ }^{1}$ Department of Ecology and Evolution, University of Chicago \\ ${ }^{2}$ Biology Department, University of North Carolina, Chapel Hill
}

\begin{abstract}
Developmental processes in multicellular organisms, and the outcomes they produce, are often evolutionarily conserved. Yet phylogenetic conservation of developmental outcomes is not reflected in functional preservation of the genes regulating these processes, a phenomenon referred to as developmental system drift (1, 2). Little is known about the evolutionary forces producing change in the molecular details of regulatory genes and their networks while preserving development outcomes. Here we address this void in knowledge by systematically swapping the Drosophila melanogaster coding and noncoding regions of the essential gap gene, giant, a key regulator of embryonic pattern formation, with orthologous sequences drawn from both closely and distantly related species within the genus. Employing sensitized genetic complementation assays, the loss of a transgene's ability to restore viability occurs across phylogeny at every interspecific level of comparison and includes both coding and noncoding changes. Epistasis is present as well - both between coding and noncoding sequences and, in a dramatic example of change-of-sign epistasis, between the only two coding substitutions separating two very closely related species. A continuous process of functional divergence hidden under conserved phylotypic developmental outcomes requires reconsideration of the prevailing view that the essential genes in conserved regulatory networks are protected from the driving forces of evolutionary change.
\end{abstract}

Correspondence: mkre@uchicago.edu

\section{Introduction}

The preservation of molecular function is a universal theme in the evolution of life, evident in the myriad of recognizably conserved molecules, proteins, genetic pathways and biochemical processes across phylogeny. All multicellular organisms, for example, possess a shared set of Hox genes regulating cell differentiation and development (3). Conserved molecular and gene expression phenotypes are believed to reflect intricately buffered developmental pathways that constrain functional evolution of member genes and circuits (4, 5). Support for this view is dominated by experiments emphasizing partial activity or replaceability of a Drosophila gene with transgenes carrying orthologs from species as distant as chicken or even human (6-9). Yet, these orthologs (13 instances in total) never fully rescue the mutant phenotypic, and they also do not restore viability (Table S1). Conservation of developmental outputs might belie functional changes in molecules that govern those outputs $(10,11)$.

Instances of this tension is apparent in the Drosophila gap gene network, a set of exquisitely studied transcription factors expressing early in embryonic development to orchestrate the highly conserved process of insect pattern formation (12). Spatio-temporal expression of the gap genes are remarkably conserved across Drosophila phylogeny, measured at nuclear resolution in three dimensions and time (13). So too is the cis-regulatory output of the pair-rule gene evenskipped, a primary target of the gap genes. When placed in D. melanogaster, eve enhancers from species in family Sepsidae, a sister group to Drosophila, respond to D. melanogaster gap proteins by driving pair-rule stripe expression nearly identically to the native eve expression pattern $(14,15)$, this despite extensive rearrangement of the relevant transcription factor binding sites. In contrast, other insect taxa, including mosquitos and moth fly, employ different maternal genes to establish head-to-tail polarity (16). In the scuttle fly, the initiation and expression of the gap genes are, moreover, quantitatively different than Drosophila, though the embryos converge to a similar developmental phenotype (17, 18).

These scattershot observations underscore the lack of a mechanistic basis for interpreting developmental system drift and highlight the need for careful systematic measurements of regulatory gene functional divergence across a phylogeny. Do these genes evolve? Is functional divergence compartmentalized to changes in cis-regulation, or do the transcription factors evolve as well? And, if so, what is the evolutionary timescale (and phylogenetic consistency) of change? We focused our experimental investigation on the gap gene giant ( $g t$ ) across six Drosophila species whose phylogenetic ancestries range from about 1 million years ago (MYA) to about 40 MYA (19, 20). The Giant protein (Gt), a basic leucine zipper transcription factor, is among the earliest proteins expressed zygotically in the blastoderm Drosophila embryo to establish landmarks for anterior-posterior patterning and segmentation $(21,22)$. Its role as a gap gene is conserved over 350 million years of divergence in Oncopeltus (23), and its DNA-binding domain remains extensively conserved in Drosophila (Fig. S1) and across bilateria evolution (24). Here we document the pace of giant functional divergence in Drosophila, both for coding and noncoding regions of the locus, and provide a mechanistic framework for understanding developmental system drift - how a regulatory network can evolve at the molecular level while maintaining a conserved system output. 


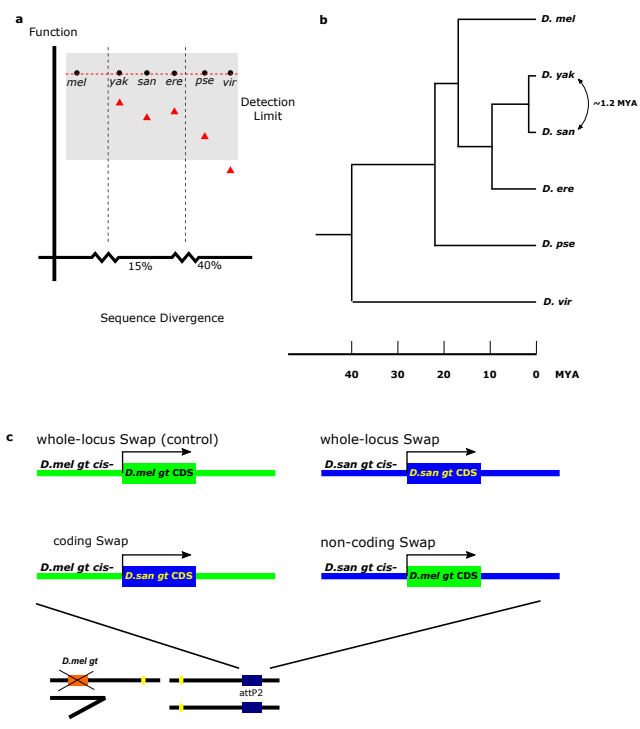

Figure 1. Approach to testing for functional divergence of $g t$ transgene orthologs. a, Competing hypotheses: Functional stasis (black dots) - gt orthologs will be indistinguishable; Functional divergence (red triangles) - gt orthologs diverge and will be distinguishable if experimental design has sufficient resolution. In this example, only vir gt functional divergence is detectable (shaded region depicts limits of experimental resolution). Vertical dashed lines mark sequence divergence relative to mel (Methods). b, Phylogenetic relationship of species investigated. c, Site-specific phiC31 transgenesis using whole-locus and chimeric giant.

\section{Results}

Experimental approach: We employed phiC31 site-specific genetic transformation (25) to study the phenotypic output of giant alleles from different species when placed in $D$. melanogaster (mel) (Fig. 1). We generated gt whole-locus genotypes carrying sequences orthologous to the $27 \mathrm{~kb}$ native locus - an interval that restores viability in a complementation assay with the mel control transgene (26) (Fig. 1b, c). We also generated interspecies transgene chimeras by swapping with the mel whole-locus sequence either the protein coding or the noncoding region from each of the five other species (Table S2; a total of 30 transgenic lines). The fluorescent protein eGFP is commonly appended to proteins as a tag to visualize their cellular distribution and function (27). As a means for amplifying possible functional differences among $g t$ proteins, we added, in a parallel set of transgenes, an eGFP carboxy-terminal tag to our whole-locus and chimeric transgenes. We scored the relative viability (hereafter RV) - defined as the ratio of $\mathrm{F} 1$ flies carrying either an interspecies or control transgene, identified with fluorescent eye markers in the offspring of test crosses carrying a null allele $\left(g t^{\mathrm{X} 11}\right)$ at the native locus (Fig. S2, S3). We measured RV in both male and female separately, anticipating that transgene restoration of viability by $g t$ orthologs might differ in the two sexes (26). We also sensitized our RV measurements by analyzing flies carrying a single copy of the $g t$ transgene.

Functional divergence of distant orthologs: We first investigated the $g t$ ortholog from $D$. virilis (vir), the most distant relative in the genus to $m e l$ (Fig. 1b; common ancestor 40 MYA (19)). Whole-locus RV is significantly reduced (RV $=0.56$ ) in males and is essentially lethal in females (Fig 2a, $\mathrm{d}, \mathrm{g})$. The vir coding region alone restores full $\mathrm{RV}$ in both sexes; vir noncoding sequences restores full RV in males but reduces RV significantly in females, though not to lethality $((\mathrm{RV}=0.21)$; Fig $2 \mathrm{f})$. The lethality driven by whole-locus vir in females, therefore, requires epistatic contributions from the vir noncoding and coding regions (Fig 2f). A vir coding contribution to loss of RV is confirmed by the eGFP-tagged version of vir coding ( $R V=0.52$; Fig $2 \mathrm{~h}$ ). We also observed a skewed sex ratio in adults when endogenous $g t^{m e l}$ is replaced by two copies of $g t^{v i r}$ (male:female $=3.68: 1$, Table S7). Collectively, these results identify functional differences in both coding and noncoding regions and reveal epistasis for RV between the two regions.

Next, we investigated the $g t$ ortholog of $D$. pseudoobscura (pse). This species is estimated to share a common ancestor with mel around 20 MYA (19), half the time separating $m e l$ from vir (Fig. 1b). Carriers of the $g t^{p s e}$ wholelocus ortholog exhibits reduced RV in both males and females, though to a lesser extent than carriers of $g t^{v i r}$ (Fig 2a, d). The reduction in RV by $g t^{p s e}$ is largely attributable to noncoding sequence (Fig 2f), and, like $g t^{\text {vir }}$, there is also a coding contribution. Specifically, whereas the $g t^{p s e}$ coding shows reduced RV with its $g t^{p s e}$ noncoding region, the chimera carrying a $g t^{\text {mel }}$ coding region does not (Fig 2a, c; Table S5). The set of experiments with pse and vir show striking parallels: strong contributions to reduced RV by the noncoding region; a contribution by the coding regions; and epistatic interaction between coding and noncoding for RV for $g t^{v i r}$ and possibly for $g t^{p s e}$ (Fig 2a,c).

Functional divergence of closely related orthologs: $D$. yakuba (yak), D. santomea (san) and D. erecta (ere) belong to the same phylogenetic clade that has a common ancestor with mel 10 MYA (19) (Fig. 1b); one of them, san, produces viable hybrids with mel. Reduced RV is observed in two of the three species: $s a n-g t^{\text {san }}$ whole locus (Fig 2d), $g t^{\text {san }}$ eGFP-tagged whole locus, and $g t^{\text {san }}$ coding-only (Fig. 2g, h); and ere $-g t^{\text {ere }}$ eGFP-tagged whole locus. Thus, even on the relatively short timescale of $10 \mathrm{MY}$ separating this clade of species from mel, the experiments functionally distinguish their $g t$ alleles from the mel ortholog.

Species hybrids: Our viability assays thus far reveal functional differences between the mel allele and the san, ere, pse and vir gt orthologs. Unresolved is whether yak, the remaining species in our $g t$ analysis, might also have functionally diverged from $\mathrm{mel} g t$, albeit more subtly. We investigated this question with species hybrids. Crosses between mel females and san males produce sterile hybrid female progenies only. We have recently shown that $\mathrm{mel} \mathrm{Gt}$, differing by seven amino acid substitutions from san Gt (Table S4), causes reduced female viability in the hybrid (28). Acting on this finding, we tested additional $g t$ transgene orthologs in $\mathrm{mel} / \mathrm{san}$ hybrids by crossing mel females hemizygous for a transgene to san males (Fig. S4). In this cross, RV is estimated from the number of hybrid F1 flies carrying either the $g t$ transgene or a control chromosome bearing no transgene. Chimeric transgenes whose coding regions have been replaced by yak, ere or san orthologs, under the regulatory control of mel noncoding region, all eliminate the deleterious effect of $\mathrm{mel} \mathrm{Gt}$ in hybrid 
a)

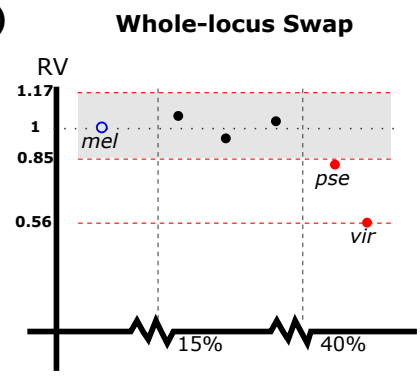

d)

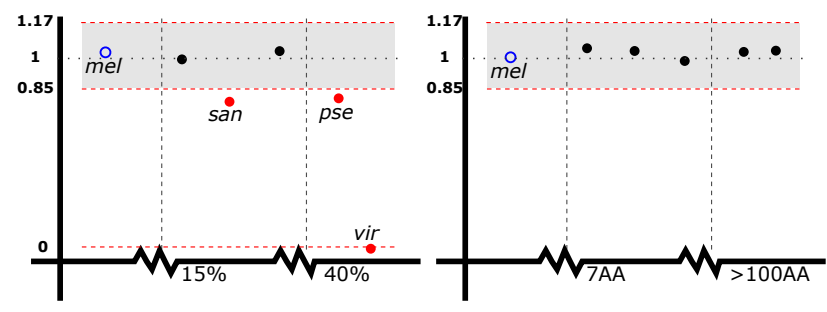

g) eGFP-tagged Whole-locus Swap

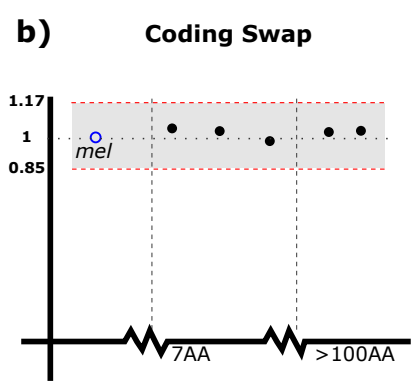

\section{c) Non-coding Swap}

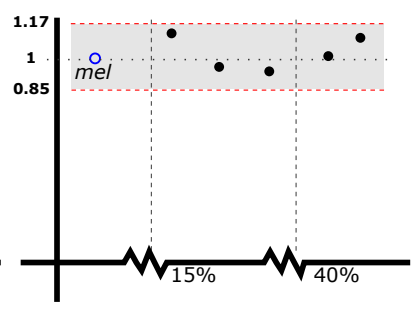

f) Non-coding Swap

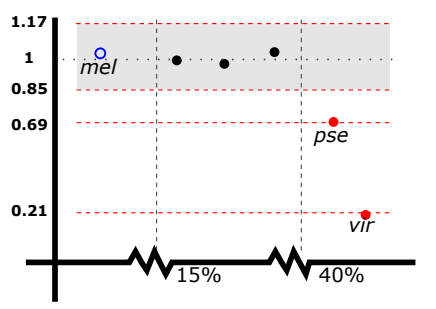

i)

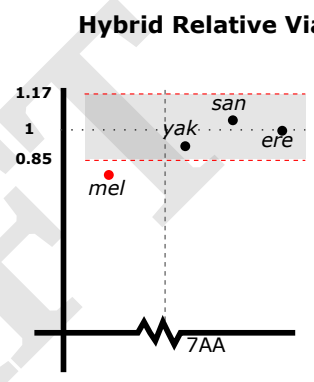

Figure 2. Complementation assays reveal extensive functional divergence across Drosophila phylogeny. Single copy transgene rescue in males (a-c) and females (d-h). d, Whole-locus vir gt restores mel female viability at a low rate $(<0.2 \% \mathrm{RV})$. g-h, Female RV using eGFP-tagged $g t$ transgenes. Whole-locus eGFP-tagged vir gt restores mel female viability only when two copies are present. $\mathbf{i}, \mathrm{mel}$ gt coding region is deleterious in mel/san hybrids. For a-h, the species order is (left-right) mel, yak, san, ere, pse and vir. RV values significantly different from mel are labeled in red. For illustrative purpose, shaded region represents $80 \%$ power to detect at $p<0.05$ a $15 \%$ difference in viability between control and experimental transgene (e.g., RV=0.85) for a sample of 1240 adults (see Methods). Sample sizes are given in Table S5.

females (Fig 2i). In this sensitized hybrid genetic environment, we are thus able to place the functional divergence in the protein leading to reduced hybrid viability caused by mel Gt to changes on the phylogenetic branch leading to mel itself.

Functional divergence of the san $g t$ protein: Transgenic yak and san $g t$ proteins under the regulatory control of mel noncoding sequence have significantly different RV in mel (Fig. 2h). This means that our experiments have detected $g t$ functional divergence at every timescale separating the six species employed in our analysis. san and yak have a common ancestor estimated to be only 1.2 MYA (20), and $\mathrm{Gt}^{\text {yak }}$ and $\mathrm{Gt}^{\text {san }}$ proteins differ by only two substitutions - A351V and $+4 \mathrm{Q}-$ (Fig. 3a). Gt ${ }^{y a k}$ is identical at both sites to the allele in $D$. teissieri, the closest outgroup species, indicating that the $\mathrm{Gt}^{y a k}$ carries both ancestral states (Fig. S3). We confirmed that the yak and san Gt alleles used in this experiment are both common alleles, not unique to specific populations of either species (Fig. S5, S6). With only two substitutions, both of which occurred in san, there are only two possi- ble intermediate evolutionary paths. We investigated both of them in mel with eGFP-tagged transgenes carrying the two single-substitution genotypes under the regulatory control of mel noncoding sequence. Our RV assay reveals a significant increase in RV for the $+4 \mathrm{Q}$ substitution alone and a significant decrease for the A351V substitution alone. Together, the two substitutions produce the most severe decrease in RV (Fig. 3b). Thus, both single substitutions have significant RV effects, the two possible trajectories differ significantly, and there is sign epistasis along one path. To summarize, no individual substitution in this sensitized experimental system is functionally inert, and together the two substitutions interact at the level of RV.

\section{Discussion}

Our experimental results reveal a continuous process of functional divergence across Drosophila phylogeny and timescales, a sharp refutation of interchangeability of genes regulating evolutionarily conserved developmental processes. Our sensitized assays, employing appropriate 
bioRxiv preprint doi: https://doi.org/10.1101/2021.07.08.451553; this version posted July 9, 2021. The copyright holder for this preprint (which was not certified by peer review) is the author/funder, who has granted bioRxiv a license to display the preprint in perpetuity. It is made available under aCC-BY-NC-ND 4.0 International license.

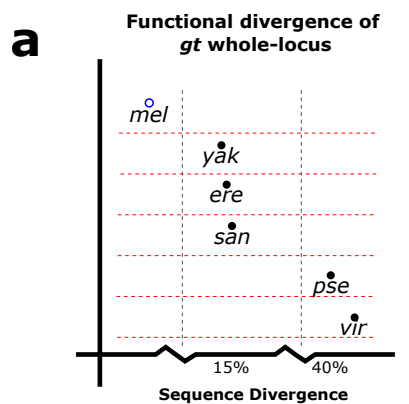

b

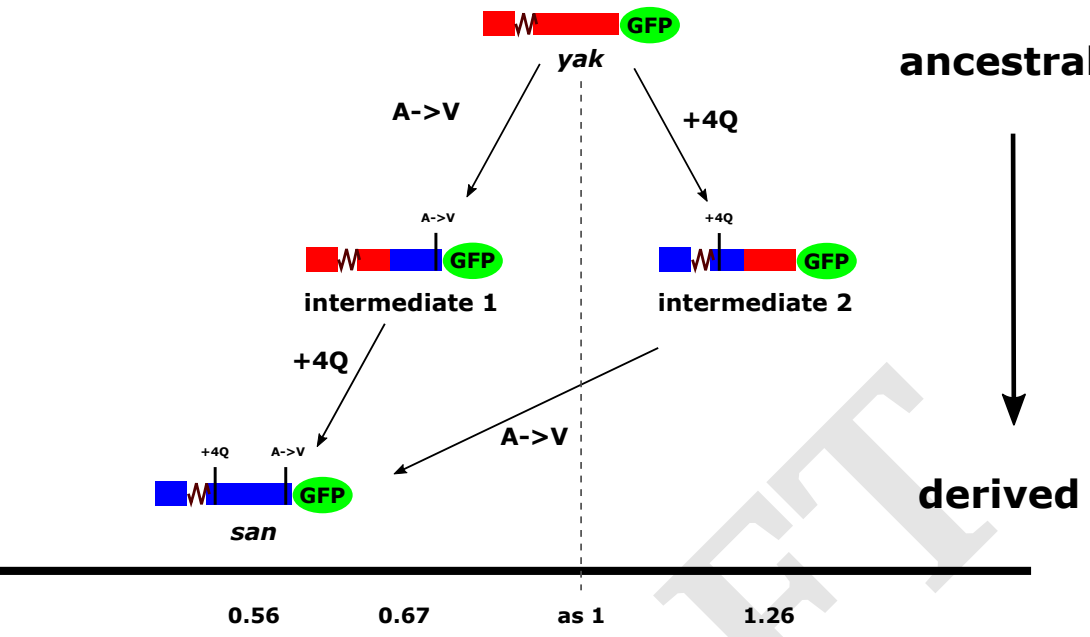

Relative Viability (in mel background)

Figure 3. The two possible evolutionary intermediates of gt coding substitutions between yak and san have distinct (and opposite) relative viability effects in mel. a, Summary of $g t$ functional divergence inferred from whole-locus or chimeric transgenes. Horizontal dashed lines demarcate distinguishable orthologs. b. Reconstructed ancestral states of yak/san gt protein and alternative evolutionary paths of divergence. Both alternative trajectories require non-neutral intermediates. Data in Table S6.

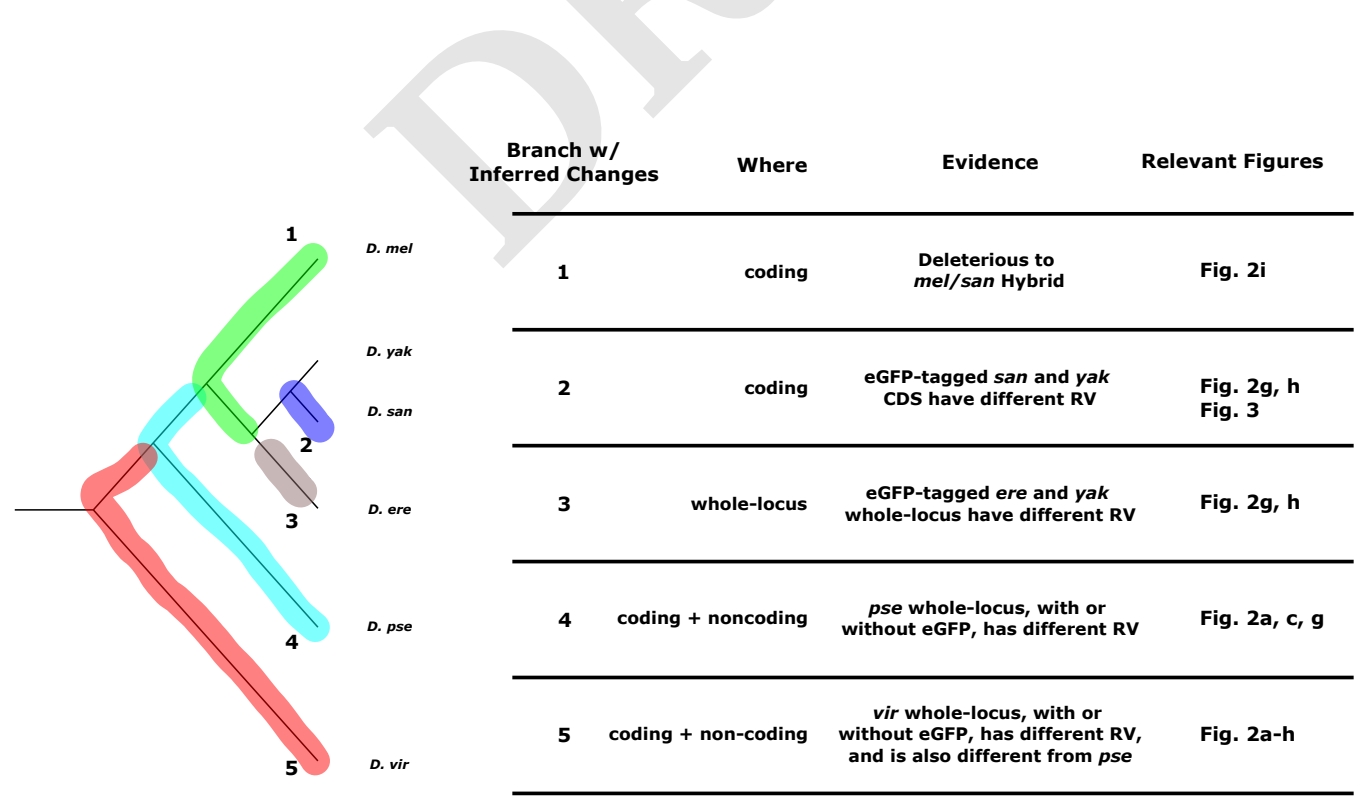

Figure 4. Continual functional divergence of the gt locus in Drosophila. A parsimonious reconstruction of functional changes in $g t$ mapped onto phylogenic branches, marked by different colors. Experimental evidence for viability differences attributable whole-locus or chimeric transgene divergence is listed alongside each branch. Functional divergence in noncoding regions inferred from species-specific epistasis is not included because of uncertainty about branch assignment. 
transgene controls inserted into the same chromosomal docking site, identified functional divergence attributable to both coding and noncoding regions along nearly every branch of the phylogeny (Fig 4). Most published studies of orthologous gene function (see Table S1), i.e., interchangeability, investigate only protein coding regions and not the whole locus. Our study reveals limitations in this approach: none of protein coding regions alone from any of the five species significantly reduced RV when driven by the mel noncoding region (Fig. 2b, e). Our finding points to the whole locus as an integral target for $g t$ functional evolution.

Functional changes in $g t$ are relevant to understanding the genetics of interspecies hybrid incompatibility. Here, our experiments amplified on a recent finding that identified the protein coding region of $g t$ in causing inviability in hybrids between mel and san (28). We mapped those differences to substitutions in the phylogenetic branch leading to mel from

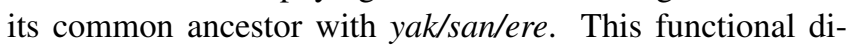
vergence is not unique to this single lineage, however, but rather is one instance of a continuous process of functional divergence across Drosophila phylogeny (Fig. 4). Hybrid incompatibility generally results from functional divergence of two interacting genes, one in each of two species, which when brought together in a hybrid, fail to function properly. The functional divergence in $g t^{\text {mel }}$ protein is, therefore, likely accompanied by similar functional divergence in one or more interacting partners in san. In a search for a partner to $g t$, we discovered that orthologs of the gap gene tailless from mel and san, like $g t$, also differ in their effects on viability in the hybrid (28). In a broader context, continuous functional evolution of $g t$, as documented here, may be representative of other "conserved" genes in the gap gene network, and illustrative of the process of rapid molecular evolution leading to hybrid incompatibility.

We believe our unequivocal findings in experimental assays - a rapid, continuous process of $g t$ functional evolution in coding and noncoding regions - are relevant to understanding population genetic mechanisms governing $g t$ evolution. In general, one expects natural selection to be many orders of magnitude more sensitive to the fitness effects of subtle functional changes than those that can be measured in our laboratory experiment. In this context, no organism has received more attention than Drosophila in a quest to understand the extent of adaptive evolution driving gene and genome evolution, and there is now near-universal agreement that natural selection is the predominant driving force in these large-population-size species (29, 30). Our findings suggest that the very same mechanism - natural selection - may be responsible for the continuous pace of $g t$ functional evolution. Especially illuminating are the two coding substitutions between the very closely related species yak and san: both evolutionary intermediates, and the combination of substitutions together, all exhibit significant viability effects in a mel genetic background. The fact that these $g t$ intermediates are distinctly different in the same genetic background suggests to us that natural selection is likely to have been involved in their substitution, even if the fitness effects are smaller in their ancestral backgrounds.

Under this mechanistic framework, the conservation of gap gene network output is achieved both by selective constraint acting on the network, as well as by a continuous process of functional refinement to individual genes and their cross-regulatory interactions. The continuous functional divergence of gap genes also gives rise, inevitably, to changes in the detailed molecular mechanisms by which the network directs pattern formation, a characteristic of developmental system drift. Our discovery of rapid functional divergence of $g t$ requires reassessment of the tempo and mode of molecular evolution of regulatory genes belonging to conserved developmental systems.

\section{ACKNOWLEDGEMENTS}

This work was supported by the National Science Foundation under award 1916895 to M.K., and by NIGMS R01 GM125715 to D.R.M. We thank John Reinitz, David Stern, Urs SchmidtOtt, Manyuan Long and Andreas Wagner for comments, and the University of Chicago DNA sequencing \& Genotyping Facility for Sanger sequencing.

\section{AUTHOR CONTRIBUTIONS}

W.C. and M.K. conceived the project and shared in writing the manuscript. W.C. performed the transgenic and viability experiments and carried out the analysis. D.R.M. shared in discussions about the project, provided $D$. santomea stocks, gave advice about conducting the interspecific hybrid experiment, and contributed to editing the manuscript.

\section{References}

1. Sommer, R.J. Evolution of regulatory networks: Nematode vulva induction as an example of developmental systems drift. Advances in Experimental Medicine and Biology, 751:79-91, 2012. ISSN 00652598. doi: 10.1007/978-1-4614-3567-9_4.

2. True, J.R. and Haag, E.S. Developmental system drift and flexibility in evolutionary trajectories. Evolution and Development, 3(2):109-119, mar 2001. ISSN 1520-541X. doi: 10.1046/j.1525-142x.2001.003002109.x.

3. Akam, M. Hox genes and the evolution of diverse body plans., sep 1995. ISSN 09628436.

4. Masel, J. and Siegal, M.L. Robustness: mechanisms and consequences, sep 2009. ISSN 01689525.

5. Geiler-Samerotte, K., Sartori, F.M. and Siegal, M.L. Decanalizing thinking on genetic canalization, apr 2019. ISSN 10963634.

6. Lutz, B., Lu, H.C., Eichele, G., Miller, D. and Kaufman, T.C. Rescue of Drosophila labial null mutant by the chicken ortholog Hoxb-1 demonstrates that the function of Hox genes is phylogenetically conserved. Genes and Development, 10(2):176-184, jan 1996. ISSN 08909369. doi: 10.1101/gad.10.2.176.

7. Nagao, T., Leuzinger, S., Acampora, D., Simeone, A., Finkelstein, R., Reichert, H. and Furukubo-Tokunaga, K. Developmental rescue of Drosophila cephalic defects by the human Otx genes. Proceedings of the National Academy of Sciences of the United States of America, 95(7):3737-3742, mar 1998. ISSN 00278424. doi: 10.1073/pnas.95.7.3737.

8. Halder, G., Callaerts, P. and Gehring, W.J. Induction of ectopic eyes by targeted expression of the eyeless gene in Drosophila. Science, 67(5205):1788-1792, 1995. ISSN 00368075. doi: $10.1126 /$ science.7892602.

9. Malicki, J., Schughart, K. and McGinnis, W. Mouse Hox-2.2 specifies thoracic segmental identity in Drosophila embryos and larvae. Cell, 63(5):961-967, nov 1990. ISSN 00928674. doi: 10.1016/0092-8674(90)90499-5.

10. Barrière, A., Gordon, K.L. and Ruvinsky, I. Coevolution within and between Regulatory Loc Can Preserve Promoter Function Despite Evolutionary Rate Acceleration. PLOS Genetics, 8(9), sep 2012. ISSN 15537390. doi: 10.1371/journal.pgen.1002961.

11. Gordon, K.L. and Ruvinsky, I. Tempo and mode in evolution of transcriptional regulation, jan 2012. ISSN 15537390

12. Jaeger, J. The gap gene network, 2011. ISSN 1420682X.

13. Wunderlich, Z., Fowlkes, C.C., Eckenrode, K.B., Bragdon, M.D., Abiri, A. and DePace, A.H. Quantitative comparison of the anterior-posterior patterning system in the embryos of five drosophila species. G3: Genes, Genomes, Genetics, 9(7):2171-2182, jul 2019. ISSN 21601836. doi: 10.1534/g3.118.200953.

14. Hare, E.E., Peterson, B.K., Iyer, V.N., Meier, R. and Eisen, M.B. Sepsid even-skipped Enhancers Are Functionally Conserved in Drosophila Despite Lack of Sequence Conservation. PLOS Genetics, 4(6):e1000106, jun 2008. ISSN 1553-7404. doi: 10.1371/JOURNAL. PGEN.1000106.

15. Hare, E.E., Peterson, B.K. and Eisen, M.B. A Careful Look at Binding Site Reorganization in the even-skipped Enhancers of Drosophila and Sepsids. PLOS Genetics, 4(11):e1000268, nov 2008. ISSN 1553-7404. doi: 10.1371/JOURNAL.PGEN.1000268. 
bioRxiv preprint doi: https://doi.org/10.1101/2021.07.08.451553; this version posted July 9, 2021. The copyright holder for this preprint (which was not certified by peer review) is the author/funder, who has granted bioRxiv a license to display the preprint in perpetuity. It is made available under aCC-BY-NC-ND 4.0 International license.

16. Yoon, Y., Klomp, J., Martin-Martin, I., Criscione, F., Calvo, E., Ribeiro, J. and Schmidt-Ott, $\mathrm{U}$. Embryo polarity in moth flies and mosquitoes relies on distinct old genes with localized transcript isoforms. eLife, 8, oct 2019. ISSN 2050084X. doi: 10.7554/eLife.46711.

17. Wotton, K.R., Jimenez-Guri, E., Crombach, A., Janssens, H., Alcaine-Colet, A., Lemke, S., Schmidt-Ott, U. and Jaeger, J. Quantitative system drift compensates for altered maternal inputs to the gap gene network of the scuttle fly megaselia abdita. eLife, 2015(4), jan 2015. ISSN 2050084X. doi: 10.7554/eLife.04785.

18. Crombach, A., Wotton, K.R., Jiménez-Guri, E. and Jaeger, J. Gap Gene Regulatory Dynamics Evolve along a Genotype Network. Molecular Biology and Evolution, 33(5):1293-1307, may 2016. ISSN 15371719. doi: 10.1093/molbev/msw013.

19. Suvorov, A., Kim, B., Wang, J., Armstrong, E., Peede, D., D'Agostino, E., Price, D., Wadell, P., Lang, M., Courtier-Orgogozo, V., David, J., Petrov, D., Matute, D., Schrider, D. and Comeault, A. Widespread introgression across a phylogeny of 155 Drosophila genomes, jan 2020. ISSN 26928205.

20. Turissini, D.A. and Matute, D.R. Fine scale mapping of genomic introgressions within the Drosophila yakuba clade. PLoS Genetics, 13(9):e1006971, sep 2017. ISSN 15537404. doi: 10.1371/journal.pgen.1006971.

21. Petschek, J.P., Perrimon, N. and Mahowald, A.P. Region-specific defects in I(1)giant embryos of Drosophila melanogaster. Developmental Biology, 119(1), 1987. ISSN 00121606. doi: 10.1016/0012-1606(87)90219-3.

22. Eldon, E.D. and Pirrotta, V. Interactions of the Drosophila gap gene giant with maternal and zygotic pattern-forming genes. Development, 111(2):367-378, 1991. ISSN 09501991.

23. Liu, P.Z. and Patel, N.H. Giant is a bona fide gap gene in the intermediate germband insect, Oncopeltus fasciatus. Development, 137(5):835-844, mar 2010. ISSN 09501991. doi: 10.1242/dev.045948

24. Nitta, K.R., Jolma, A., Yin, Y., Morgunova, E., Kivioja, T., Akhtar, J., Hens, K., Toivonen, J., Deplancke, B., Furlong, E.E. and Taipale, J. Conservation of transcription factor binding specificities across 600 million years of bilateria evolution. eLife, 2015(4), mar 2015. ISSN 2050084X. doi: 10.7554/eLife.04837.

25. Groth, A.C., Fish, M., Nusse, R. and Calos, M.P. Construction of Transgenic Drosophila by Using the Site-Specific Integrase from Phage $\phi$ C31. Genetics, 166(4):1775-1782, apr 2004. ISSN 00166731. doi: 10.1534/genetics.166.4.1775.

26. Manu, Ludwig, M.Z. and Kreitman, M. Sex-specific pattern formation during early Drosophila development. Genetics, 194(1):163-173, may 2013. ISSN 00166731. doi: 10.1534/genetics.112.148205.

27. Sokolovski, M., Bhattacherjee, A., Kessler, N., Levy, Y. and Horovitz, A. Thermodynamic Protein Destabilization by GFP Tagging: A Case of Interdomain Allostery. Biophysical Journal, 109(6):1157-1162, sep 2015. ISSN 15420086. doi: 10.1016/j.bpj.2015.04.032.

28. Chang, W., Kreitman, M. and Matute, D.R. Gap genes are involved in hybrid inviability between Drosophila melanogaster and D. santomea. bioRxiv, 2021.

29. Messer, P.W. and Petrov, D.A. Frequent adaptation and the McDonald-Kreitman test. Proceedings of the National Academy of Sciences of the United States of America, 110(21), 2013. ISSN 00278424. doi: 10.1073/pnas.1220835110.

30. Sella, G., Petrov, D.A., Przeworski, M. and Andolfatto, P. Pervasive natural selection in the Drosophila genome?, 2009. ISSN 15537390. 